\title{
La fatigue du patron pêcheur dans l'activité de chalutiers pélagiques : approche d'anthropologie cognitive
}

The captain's fatigue in the pelagic trawler activity: a cognitive anthropology approach

La fatiga del capitan de pesca en la actividad de los pescadores pelágicos : un enfoque de antropologia cognitiva

Bastien Sennegon, Benoit Grison, Philippe Ravier et Olivier Buttelli

\section{OpenEdition}

Édition électronique

URL : http://journals.openedition.org/pistes/1768

DOI : $10.4000 /$ pistes. 1768

ISSN : 1481-9384

Éditeur

Les Amis de PISTES

Édition imprimée

Date de publication : 1 mai 2011

Référence électronique

Bastien Sennegon, Benoit Grison, Philippe Ravier et Olivier Buttelli, « La fatigue du patron pêcheur dans l'activité de chalutiers pélagiques : approche d'anthropologie cognitive », Perspectives

interdisciplinaires sur le travail et la santé [En ligne], 13-1 | 2011, mis en ligne le 01 mai 2011, consulté le 03 mai 2019. URL : http://journals.openedition.org/pistes/1768 ; DOI : 10.4000/pistes. 1768

Ce document a été généré automatiquement le 3 mai 2019.

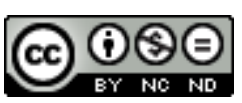

Pistes est mis à disposition selon les termes de la licence Creative Commons Attribution - Pas d'Utilisation Commerciale - Pas de Modification 4.0 International. 


\section{La fatigue du patron pêcheur dans l'activité de chalutiers pélagiques : approche d'anthropologie cognitive}

The captain's fatigue in the pelagic trawler activity: a cognitive anthropology approach

La fatiga del capitan de pesca en la actividad de los pescadores pelágicos : un

enfoque de antropologia cognitiva

Bastien Sennegon, Benoit Grison, Philippe Ravier et Olivier Buttelli

\section{Introduction}

1 Le milieu marin a souvent été considéré comme un environnement relativement éprouvant, voire hostile pour l'être humain. Encore aujourd'hui, la navigation reste tributaire de la météo et des risques que celle-ci génère. À titre d'illustration, $12 \%$ des accidents de navire de pêche entre 1997 et 2007 sont principalement consécutifs à une dégradation des conditions météorologiques (BEA-mer, 2007).

De plus, le milieu maritime est aussi reconnu par Le Roy et Breuille (1997) en tant que secteur professionnel à haut risque : en milieu isolé (notion du collectif, rythmes de travail), sur un flotteur (sécurité, habitabilité, hygiène); et dans un environnement naturel difficilement maîtrisable. Minguy et Chauvin (1998) témoignent eux aussi d'un environnement de travail ardu où les gens de mer évoluent de nuit comme de jour, et sans aucune interruption.

D'après le Bureau d'enquêtes sur les événements de mer (BEA-mer, 2007), le métier de la pêche est l'un des plus dangereux dans les professions maritimes avec un taux de décès de 19,6 pour 100 000. Pour les embarcations de pêche, les causes d'accidents sont multiples : abordage (24\%), échouement (13\%), croche (10\%), homme à la mer (7\%)... Ainsi, sur la période de 1997 à 2007, la plus forte proportion d'accidents équivaut aux abordages 
impliquant des navires de pêche. Le BEA-mer impute clairement ces accidents à la fatigue, à l'insuffisance d'effectif, et au manque de priorité accordée pour la veille nautique.

De ce fait, la relation entre l'affaiblissement du facteur humain et le risque d'avoir un accident paraît établie dans l'environnement marin. Les baisses de vigilance, notamment causées par la fatigue et/ou la privation de sommeil, pourraient être un facteur de risque dans ces métiers de la mer. L'accumulation de fatigue est vécue dans ce contexte, gérée au quotidien par des marins qui ne comptent pas leurs heures hebdomadaires de travail, tant ces dernières sont nombreuses.

Il apparaît que la pêche est en première ligne du point de vue des événements de mer. En effet, la Direction générale de la mer et des transports (2007) a répertorié 3400 accidents du travail maritime pendant cette année, dont 1682 accidents du travail pour la pêche, soit 121 accidents pour 1000 .

6 La fatigue pourrait donc être un facteur de risque pour les navires de pêche. Les enjeux de sécurité paraissent étroitement liés aux conditions de travail et à l'accumulation de fatigue des gens de mer. Cette problématique est sous-tendue par des liens multilatéraux entre le contexte d'activité harassant, les facteurs de risque et ceux de sécurité.

\section{Cadre théorique}

7 La fatigue s'avère complexe d'un point de vue théorique, car elle englobe différentes notions, qui sont elles-mêmes en interrelation :

- les rythmes biologiques (Benoit et Foret, 1992 ; Saper et coll., 2005), notamment avec le sommeil (Jouvet, 2000 ; Siegel, 2002) et la vigilance (Boivin et coll., 2005 ; Itti et coll., 2005 ; Lavoie et coll., 2003) ;

- le stress (Cespuglio et coll., 2000 ; Chrousos et Gold, 1995);

- ou encore la charge de travail dans le domaine de l'ergonomie (Jourdan et Theureau, 2002).

Ces différentes notions sont toutes plus ou moins floues, leurs contours ne sont pas strictement délimités, alors même que la fatigue reste toujours un concept problème sur le terrain. On peut à cet égard établir un parallèle avec l'ouvrage sur la charge mentale coordonné par Jourdan et Theureau (2002) qu'ils avaient eux-mêmes caractérisée de " notion floue mais vrai problème ».

9 Parmi les études réalisées dans le milieu maritime, nous pouvons déjà repérer une certaine hétérogénéité au travers des notions susmentionnées puisqu'elles recoupent différents champs scientifiques, de la physiologie à la psychologie. En effet, nous pouvons aborder aussi bien l'état de vigilance des marins à partir d'électro-encéphalogrammes (La Giclais, 2009), qu'à partir des perceptions subjectives d'un individu (Gander et coll., 2008 ; Stampi, 1989 ; Tirilly et Foret, 2000). Il en est de même pour le stress qui peut s'évaluer à travers des dosages hormonaux (Gunnarsson et coll., 2004), mais également sous sa forme perçue de manière individuelle (Le Scanff, 2000). La charge de travail présente pareillement des aspects tantôt physiologiques, tantôt psychologiques. Il semblerait que la fatigue se situe à l'intersection de ces notions imprécises et hétérogènes.

Ainsi, la fatigue globale d'un individu se caractériserait non seulement d'un point de vue physiologique, mais également d'un point de vue psychologique. Dans cette étude nous allons essentiellement nous focaliser sur l'aspect perçu de la fatigue, à travers les perceptions et l'expérience vécue des marins. 
11 Dans le domaine de l'ergonomie, l'activité du sujet est majoritairement étudiée dans l'environnement naturel/culturel du sujet (Grison et Riff, 2002 ; Leplat, 2008). Ce critère est donc à prendre en considération dans l'élaboration de l'observatoire, afin de construire une méthodologie qui respecte les contraintes de l'environnement (Leplat, 2002 ; Theureau et Jeoffroy, 1994).

12 Les outils destinés à recueillir ces données subjectives doivent être objectivés. Sur le plan psychologique, Leplat (2008) et Theureau (2006) conseillent de combiner plusieurs méthodes, afin de recueillir différents ordres de données et de pouvoir les recouper entre elles.

13 L'observation directe est un outil régulièrement utilisé en ergonomie (Leplat, 2000, Theureau, 2006). Il semble incontournable dans l'environnement maritime pour avoir accès au vécu des individus dans leur activité (Maline et Dorval, 1991a; 1991b ; Hazelhurst, 1999). Pour autant, l'observation ne suffit pas au recueil de données subjectives sur la fatigue en milieu maritime, car elle n'ouvre pas l'accès aux perceptions de l'individu au cours de son activité (Theureau, 2004). De ce fait, il est important d'y associer un recueil de données sous forme de verbalisations (Leplat, 2000 ; Vermersch, 2000). Ces entretiens individuels permettent une forme de prise de conscience de l'individu à propos de la tâche (Vermersch, 2000). L'observation? de la fatigue perçue se structurerait donc autour de ces deux outils complémentaires que sont l'observation directe et les entretiens individuels.

\section{Méthodologie}

\subsection{Les marins pêcheurs étudiés}

14 Les marins pêcheurs de cette étude sont des "patrons » de chalutiers pélagiques (principe du chalutage à profondeur variable impliquant deux bateaux, le "bœuf » qui dirige les manœuvres, et le "veau ", espacés d'environ 150 mètres). Ils sont spécialisés dans la pêche à l'anchois. Les chalutiers demeurent en mer durant une semaine avec sept hommes à bord en moyenne. Le choix du poste étudié fut effectué en fonction de sa durée de travail. En effet, selon le rapport "Sécurité et conditions de travail à la pêche artisanale et semi-industrielle » (Andro et coll., 1987), le patron est la personne du bord qui prend le moins de temps de repos. Cependant, la modernisation, l'automatisation, ainsi que les récentes innovations technologiques contribuent à limiter largement les interventions physiques du patron pêcheur. Son activité se caractérise plutôt par une vigilance permanente depuis son poste de passerelle, associée aux responsabilités de gestion organisationnelle d'un navire de pêche et de son équipage.

Les patrons pêcheurs étudiés sont basés dans le port de La Turballe (France) :

- Baptiste, âgé de 33 ans, navire mesurant 24 mètres, équipage embarqué de six hommes plus un stagiaire ;

- Brice, âgé de 32 ans, navire mesurant 23 mètres, équipage embarqué de six hommes ;

- Benjamin, âgé de 41 ans, navire mesurant 22 mètres, équipage de six hommes plus un stagiaire.

Ils disposent tous les trois de leur propre paire de bateaux («bœuf» et «veau»), Benjamin ne possédant que $50 \%$ du « veau ». Les chalutiers de Brice et de Benjamin ont 
été construits il y a une quinzaine d'années, celui de Baptiste étant plus récent (quatre ans). h/24h) :

- avec Baptiste, l'observateur était à bord du 30/05 (départ de La Turballe) au 03/06 (arrivée à Saint-Jean-de-Luz); il a réembarqué du 25/10 au 27/10, la semaine se trouvant raccourcie pour cause de mauvaises conditions météorologiques (départ et arrivée du port de La Turballe);

- avec Brice, l'observateur était à bord du 05/06 au 10/06 (départ et arrivée sur Saint-Jean-deLuz) ; puis du 31/10 jusqu'au 05/11 (départ et arrivée de La Turballe) ;

- avec Benjamin, l'observateur était à bord du 12/06 (départ Saint-Jean-de-Luz) au 17/06 (arrivée à La Turballe) ; puis du 14/11 au 18/11 (départ et arrivée de La Turballe).

Simultanément à l'activité des marins, l'observateur a répertorié sur un carnet ethnographique ses observations, analyses personnelles et discussions variées. En ce qui concerne son déroulement, l'observation directe se décline en plusieurs étapes (Bachelor et Joshi, 1986 ; Becker, 1958) :

1. description systématique des phénomènes et des informations sur l'organisation;

2. évaluation de la récurrence des phénomènes observés et leur fréquence d'apparition;

3. construction des catégories d'analyse pour structurer l'organisation générale de la situation (cette phase appartient déjà en partie au traitement des données).

Pendant ces six semaines de terrain, l'observateur a mené des observations, mais également conduit des entretiens. Nous pouvons différencier deux types d'échanges :

- les «entretiens ethnographiques", informels, rentrant dans le cadre de l'observation directe ; 
- les «entretiens compréhensifs » proprement dits, avec prises de rendez-vous préalables, durées de discussion contrôlées, et enregistrement des données.

\subsubsection{Les entretiens} récurrents de l'activité des marins. Puis, en effectuant une comparaison entre les catégories de signification relatives aux différents sujets, nous avons fait ressortir des similitudes interindividuelles.

\section{Résultats}

\subsection{Description de l'activité du patron pêcheur}

\subsubsection{L'espace de travail}

L'activité du patron pêcheur se réalise sur deux niveaux distincts de l'embarcation :

- le pont supérieur qui est en partie ouvert, avec en son centre une passerelle fermée, et qui comprend le poste de pilotage (ainsi que la cabine du patron pour Benjamin) ;

- le pont intermédiaire, nommé « pont principal ", qui est couvert (ouverture sur l'arrière), avec des volumes fermés (la cabine du patron, la cuisine, le « carré » ou réfectoire, l'atelier, et une réserve).

Perspectives interdisciplinaires sur le travail et la santé, 13-1 | 2011 
Le patron passe la majorité de son temps au poste de pilotage. Sur le cumul de deux heures de pêche nous relevons une moyenne entre les trois sujets de 104 minutes à la passerelle, 10 minutes sur le pont supérieur, et 6 minutes sur le pont principal. Il ne descend que pour manger (sauf en situation de pêche où il déjeune à la passerelle), se reposer dans sa cabine, aider momentanément les matelots (démêler les câbles, modifier des réglages sur le filet), ou encore regarder fugacement l'« aspect général » du poisson.

\subsubsection{Les différentes tâches}

1 Le patron exécute de multiples tâches (cf. tableau 1) :

- il pilote son bateau et dirige la paire de chalutiers, parfois dans un trafic très dense, avec la nécessité d'anticiper sur les déplacements de chaque navire ;

- il optimise les réglages du filet en fonction de la détection des bancs d'anchois et du relief du fond ;

- il organise les différentes manœuvres à bord, dont celles en synchronisme avec le veau ;

- il planifie et dirige le débarquement des caisses d'anchois à quai.

La dégradation des conditions climatiques (accroissement de la vitesse du vent et de la hauteur de la houle) complique considérablement les différentes manœuvres.

Verbalisations 1

Baptiste: «Par mauvais temps tu vas être fatigué, il faut faire attention aux accostages. » B6 (B : Retranscription des entretiens de novembre)

Brice : «Quand il y a du mauvais temps, si tu veux filer [suivre] un cap quand il y a du poisson sur le fond, eh bien, si tu es en travers à la lame, tout chavire derrière ! [...] Et puis les manœuvres c'est plus compliqué. » B12

Benjamin : «Pour les manœuvres d'accostage, c'est plus délicat. Bon un chalutier, il peut y avoir 50 nœuds de vent, un bateau dans nos tailles, il peut rester en pêche sans souci, sans problème! Nous on rentre, parce que nous, il faut être à dix mètres l'un de l'autre pendant un quart d'heure. Même par mauvais temps ! » B32

Tableau 1. Description de l'activité du patron lors du chalutage « en bœufs »

Recherche, détection du poisson (route) : le patron est à la barre dans la passerelle, il scrute incessamment les écrans des sondeurs, et demeure en communication permanente avec les autres bateaux (VHF, CB, Fax...).

Filage, puis pêche : le train de pêche est filé, le veau se rapproche pour récupérer le bras de la fune (câble) envoyé à l'aide d'une touline (nœud lestant l'extrémité d'un cordage), puis les deux navires règlent leur vitesse et leur écartement de façon optimale. Lors du chalutage, le patron focalise son attention sur les "tâches qui rentrent" (netzsonde, sonde qui flotte au dessus de l'ouverture du filet), il modifie sa route et la profondeur du chalut selon de multiples Au cours des étapes de informations (trafic, nature $\mathrm{du}$ fond, heure de la journée, données filage et virage, le patron provenant $\mathrm{du}$ sondeur ou du sonar, communications diverses des autres bateaux...).

doit diriger son navire et surveiller simultanément
par l'arrière les manœuvres du train de pêche. 
Virage : le train de pêche est viré après avoir récupéré le bras de fune au cours du rapprochement avec le veau par l'envoi d'une touline, le chalut viré sur l'enrouleur (le patron donne des coups de barre en zigzaguant pour effectuer cette opération proprement), la pochée est hissée à bord à l'aide de la caliorne (treuil pour hisser le filet à bord) afin d'être déversée dans les parcs. Le patron descend immédiatement pour vérifier par lui-même la quantité (pourcentage en fonction du tonnage) ainsi que la qualité (aspect et taille) du poisson.

Route, triage du poisson : le patron est à la barre pendant que les matelots séparent l'anchois des autres poissons ; dès que possible, le patron descend quelques instants pour les aider ; dans ce cas, le radar et la surveillance visuelle à l'aide des jumelles sont d'une importance capitale.

Repos : le patron est dans sa cabine, allongé dans sa couchette, le bateau peut être en route ou stoppé moteur débrayé.

Synthèse réalisée à partir du carnet d'observation.

\subsubsection{La charge de travail}

Cette charge se décline en trois activités distinctes réalisées conjointement par le patron : le pilotage, la surveillance, et la communication (cf. tableau 2).

Tableau 2. La charge de travail du patron

\begin{tabular}{|l|l|l|l|}
\hline & Communications & Surveillance & $\begin{array}{l}\text { Pilotage / manœuvre du } \\
\text { train de pêche }\end{array}$ \\
\hline Brice & $\begin{array}{l}\text { minutes } \\
-4 \text { envois par fax }\end{array}$ & $\begin{array}{l}7 \text { utilisations des } \\
\text { jumelles } \\
-9 \text { sorties sur le pont } \\
\text { supérieur } \\
-2 \text { descentes sur le pont } \\
\text { principal }\end{array}$ & -5 actions soit 9 minutes \\
\hline Benjamin & -17 appels CB/VHF soit 36 & $\begin{array}{l}\text { minutes } \\
\text { mumelles } \\
-7 \text { sorties sur le pont } \\
\text { supérieur } \\
-5 \text { descentes sur le pont } \\
\text { principal }\end{array}$ & -12 actions soit 15 minutes \\
\hline
\end{tabular}

Observations à travers deux situations de chalutage entre $18 \mathrm{~h}$ et $20 \mathrm{~h}$ les 08/06/04 et 16/06/04

34 L'activité de pilotage inclut toutes les manœuvres propres au navire et au train de pêche décrites précédemment (tableau 1).

L'activité de surveillance s'établit à partir des écrans disposés autour du patron pêcheur, selon leur fréquence d'utilisation. Par l'intermédiaire des appareils électroniques, le patron tente d'établir un contrôle sur tout ce qui se passe à proximité du 
chalutier. Il complète ce contrôle par un suivi visuel (utilisation fréquente des jumelles, sortie sur le pont supérieur - lorsqu'un objet non identifié flotte sur l'eau par exemple).

L'équipement électronique varie d'un bateau à l'autre, mais la configuration la plus succincte implique au moins cinq écrans à observer régulièrement :

- le sondeur, avec au minimum un écran divisé en deux, pour la sonde sous la coque et le netzsonde sur le chalut, ce dernier requérant toute l'attention du patron, les taches présentes à l'écran correspondant au poisson qui va rentrer dans le filet ;

- le GPS (Global Positioning System) qui donne la position, le cap et la vitesse, au nombre d'au moins deux à bord (dont l'un de secours) ;

- le logiciel de cartographie sur un ou deux écrans d'ordinateur (couplé aux GPS), il sert à tracer la route; il contient également une base de données gardant en mémoire toutes les routes précédentes, les zones de pêche, les meilleurs « traits » de chalut...

- le fax sur un écran d'ordinateur : c'est le moyen le plus confidentiel de communication à bord, l'information est transmise uniquement aux personnes désirées ;

- le radar qui donne des indications sur le positionnement des autres chalutiers, mais également sur l'écartement du chalut avec le « veau ». Le radar peut être doublé pour ne pas avoir à changer d'échelle de portée trop fréquemment, ce matériel devenant prioritaire par visibilité réduite ;

- le sonar (absent sur le navire de Brice) renseigne sur la position du poisson autour du bateau ; il est à l'origine de certains zigzags des chalutiers, le patron influant sur la barre, afin de suivre et faire rentrer dans le filet les taches correspondant aux poissons recherchés.

L'activité de communication se décompose entre l'échange et la prise d'informations. Les patrons communiquent très régulièrement avec des $C B$ (postes radios émetteurs/ récepteurs amateurs) et VHF (postes radios émetteurs/récepteurs à hautes fréquences, dont l'un reste branché en permanence sur le canal 16, de détresse). Ces communications servent notamment à révéler les coordonnées des chalutiers ou encore la quantité d'anchois pêchée. Dans la même optique que le sondeur, le radar, ou la cartographie, les postes sont doublés et même triplés pour ne pas avoir à moduler la fréquence de façon incessante (Baptiste possédait ainsi une dizaine de CB et VHF). Nous pouvons également noter la présence de téléphones satellites et portables, mais ceux-ci s'avérèrent beaucoup moins utilisés (trois à quatre fois par jour).

Le patron pêcheur reçoit une quantité substantielle d'informations (tableau 3) sur une durée temporelle extrêmement restreinte, voire simultanément.

\section{Verbalisations 2}

Baptiste : «Il faut trier, parce que des fois, il y en a qui servent à rien et d'autres qui sont importantes. » A3 (A : Retranscription des entretiens de juin)

Brice : « Tu accumules pas mal de trucs [...] à force de faire plein de trucs en même temps [...] c'est usant des fois. C'est la radio aussi, quand tout le monde parle en même temps. » A22

Brice : «Il ne faut pas louper d'informations, une information peut tout changer. Ça peut tout changer le déroulement de ta journée [...] tu vois hier, on était bien parti : après deux coups de chalut, on était dans les mieux. Et après, sur une décision ou une information, on est parti sud-est, et ça nous a mis à la bourre !» B23

Le patron pêcheur doit faire des choix pertinents dans ces informations, élaborer des stratégies de pêche, et demeurer concentré en permanence pendant des heures d'affilée : il ne peut se permettre de manquer une donnée qui pourrait s'avérer primordiale pour programmer la journée à venir ou même pour les jours suivants. Néanmoins, ces décisions sont parfois arrêtées collectivement - avec le patron du veau, mais aussi avec 
d'autres bateaux. La flottille de chalutiers pélagiques est ainsi constituée de différents sous-groupes qui travaillent en réseau, dans un souci d'efficience.

Verbalisations 3

Baptiste : «Le matelot, il va avoir plus de fatigue physique, et nous il y a beaucoup plus l'attention [...] la gestion, c'est peut-être ça le plus fatiguant. » A4

«Il y a toujours des choix à faire. C'est vrai que toute la journée, tu as une dizaine ou une quinzaine de choix à faire. » $\mathrm{A} 5$

Benjamin : "Tu te dis, il y a tant de bateaux là, et ça élargit ton champ de vision, même si tout se dit pas parce qu'il y a des clans en mer [...] tu vois les bateaux, bah moi je les repère déjà. J'essaye de voir qui c'est qui est à cet endroit-là, après je ne les quitte pas du radar. » A24

Toutefois, ces diverses prises de décisions sont sources de stress pour le patron pêcheur, celui-ci s'exerçant de façon ininterrompue sur les marins, avec un paroxysme au cours de la pêche proprement dite.

Verbalisations 4

Benjamin : "Il y a un truc qui va pas, j'analyse les choses et puis je me dis [...] je reviens toujours en arrière [...] je me dis j'aurais dû faire ça, et finalement, j'ai pas fait ça, alors que j'en serais là [...] tu vois je me dis, je l'ai écouté lui ; j'aurais pas dû, j'aurais dû écouter mon idée. » A16

Baptiste: «Il y a peut-être des moments où j'en ai un peu marre de devoir tout planifier. » A4

\subsection{L'organisation du travail à bord des chalutiers}

\subsubsection{La rémunération « à la part de pêche »}

41 L'organisation du travail est dictée par les contraintes d'une pêche à rendement maximal. Ce primat de la pêche est imputable au type de rémunération des pêcheurs. Effectivement, leur salaire va coïncider directement avec la quantité et la qualité du poisson ramené à quai pour être vendu à la criée (l'idéal : un poisson non abîmé et bien conservé). Si la pêche n'est pas conséquente, ou si par exemple le poisson est trop petit pour être vendu à certains clients (usines), le patron devra tout de même régler les coûts occasionnés par la sortie des chalutiers.

Verbalisations 5

Brice : « Tu as beaucoup d'enjeux, alors tu serais tout seul encore, tu supporterais peut-être mieux. Mais là, il y a tout le monde derrière! Il faut toujours rester sous pression, même si ton bateau est payé. » A16

"Quand tu vois que tu n'as rien trouvé, là, tu commences à penser [...] et là, t'as tes factures qui te reviennent [...] t'as tous les frais, pas mal de frais. Tu vois, moi, le bateau il m'a coûté sept millions, il y a deux ans, alors tu vois, j'en ai encore pour dix ans! » A24

"Avec les travaux et tout... là t'assures [...] parce que les trimestres, tu donnes de l'argent, l'assurance, payer le bateau, tu vois l'électricien, les mécaniciens [...] bah, tout ça, ça rentre en compte justement quand tu pêches pas... » A25

Cette pression psychologique induite par le système de rémunération «à la part » se profilerait donc derrière la plupart des préoccupations des patrons pêcheurs : outre les coûts engendrés par la pêche (p. ex. : plus de cent litres de gazole consommés à l'heure en moyenne), certains d'entre eux doivent rembourser au fur et à mesure l'achat de leur chalutier ou du veau de la paire.

Verbalisations 6

Benjamin : «En début de semaine, on aurait essayé d'assurer. Tu essayes toujours 
d'assurer tant que t'as pas payé tes frais, tant que t'as pas un minimum. Après une fois que t'as un minimum, t'es plus libre pour aller chercher si tu veux. » B34

\subsubsection{La capture de l'anchois}

Les bateaux mettent en pêche (filage du chalut) une fois rendus sur zone, et ensuite, enchaînent les «traits » de chalut tout au long de la journée (parfois, ils font route quand il n'y a plus de poisson dans le secteur). Ensuite, les navires regagnent le port le plus proche afin de débarquer les caisses d'anchois avant l'aube, de réembarquer des caisses vides et de la glace - pour ceux qui n'en produisent pas. La vente à la criée du port aura lieu le matin même.

Pourtant, toutes les journées ne correspondent pas à cette description :

- les patrons pêcheurs ne trouvent pas toujours immédiatement le poisson (détection de taches au sondeur à une profondeur précise). Cette phase de recherche peut durer de plusieurs heures jusqu'à plusieurs jours ;

- les chalutiers rentrent au port uniquement lorsqu'ils ont suffisamment de poisson dans les cales. Si le patron juge que le nombre de caisses n'est pas assez conséquent par rapport au temps de trajet nécessité pour rallier le premier port (aller/retour), il préférera vendre moins cher son poisson deux jours plus tard. En juin, le navire peut alors passer la nuit en mer, le moteur débrayé.

En ce qui concerne l'anchois, les «traits » de chalut sont extrêmement courts, puisqu'ils durent d'une heure et demie à deux heures, ce qui laisse peu de temps (environ une heure) à l'équipage entre les manœuvres de virage et de filage. Par exemple, les repas étaient souvent préparés avant le virage, mais le déjeuner s'effectuait après le filage.

Cependant, il convient de différencier le mois de juin du mois de novembre :

- lors de la période estivale où l'anchois est en surface, les chalutiers pêchent du lever jusqu'au coucher du soleil. C'est une période faste où les bancs d'anchois sont «purs» et omniprésents dans le golfe de Gascogne ;

- lors de la période automnale qui est plus critique, l'anchois se mélange avec les sardines et les chinchards, à des profondeurs conséquentes. La pêche se pratique de jour comme de nuit.

Verbalisations 7

Baptiste : «L'automne c'est toujours, on travaille plus la nuit, et puis souvent, on sait jamais parce que c'est jour ou nuit. La nuit, il y a du triage. Le mauvais temps aussi, c'est plus dur. » B9

le temps deviendra importante : parfois à la remontée suivante, le triage précédent ne sera pas achevé. La situation allant alors en se dégradant si plusieurs tonnes de poisson sont pêchées consécutivement.

Verbalisations 8

Brice: «Si tu 'pêches pur', il y a pas de triage, tu vois, sinon il y a des caisses partout. Tu vois, on rentre dans le port et il reste encore deux ou trois tonnes à trier [...] il faut trier tous les anchois un par un!!! » A19

«Je peux être fatigué avant eux, parce que souvent, je fais huit-neuf heures de recherche, et ils dorment eux les gars. Et après par contre, s'il y a du triage, eh ben, j'ai huit-neuf heures dans la vue... Là on n'est pas sur le même pied d'égalité ! » A24

Ici, Brice fait allusion à un autre inconvénient lié au triage, le patron ne pouvant être remplacé à la barre (au poste de pilotage) par un matelot pour aller s'allonger. 
Verbalisations 9

Benjamin: «T'as le triage, c'est ça qui te met dedans. Il faut trier le poisson alors que là il est pur : plus on va avancer dans la saison, plus ça va se compliquer. » A32 «C'est des coups de chalut d'une heure et puis, un coup de chalut, trois pochons de triage chacun! Et tu en as pour six heures à trier ça ! Un par un! » A36

\subsubsection{Les rythmes de travail}

Les chalutiers pélagiques partent sur une semaine du dimanche au vendredi (soit 119 heures en moyenne par semaine). Les principales causes de fatigue sont liées au rythme de travail élevé du marin pêcheur.

Verbalisations 10

Brice : « Nous, quand on pêche pas, on peut pas se reposer, et après tu pêches, il y a

du triage, tout ça, et tu ne peux pas dire à un gars de venir te remplacer. » B26

Benjamin, à propos des moments de fatigue : "On a beaucoup de mal à les gérer parce qu'on peut pas les éviter. » A28

"Quand on est en route que t'as les gars qui t'appellent « ça file devant! », aussitôt tu te lèves et on file [filer signifiant mettre le chalut à l'eau]. » A34

Le travail sur les chalutiers est rythmé par l'alternance des phases d'activité et de repos (cf. tableau 3). La vie à bord alterne les moments de travail intense - la pêche (la sirène qui prévient les matelots de la manœuvre imminente se prénomme d'ailleurs le «branle-bas ») et les périodes « au ralenti », où l'exercice est réduit - tel le retour vers le port.

Tableau 3. Rythmes de travail du patron - illustration sur 48 heures

\begin{tabular}{|c|c|c|}
\hline \multirow{3}{*}{ 01/06 } & 06H00-07H30 & Route (1h30 de repos) \\
\hline & 07H $30-22 \mathrm{H} 30$ & Pêche (15 h de travail) \\
\hline & $22 \mathrm{H} 30-00 \mathrm{H} 00$ & Route ( 1 h 30 de repos) \\
\hline \multirow{3}{*}{$02 / 06$} & $00 \mathrm{H} 00-02 \mathrm{H} 00$ & Débarquement des caisses ( $2 \mathrm{~h}$ de travail) \\
\hline & 02H00-06H0O & Route ( $4 \mathrm{~h}$ de repos) \\
\hline & 06H00-22H00 & Pêche (16 h de travail) \\
\hline \multirow{2}{*}{$03 / 06$} & $22 \mathrm{H} 00-04 \mathrm{H} 30$ & Route (6 h 30 de repos) \\
\hline & $04 \mathrm{H} 30-06 \mathrm{H} 00$ & Débarquement des caisses ( 2 h 30 de travail) \\
\hline
\end{tabular}

Observation effectuée en juin avec Baptiste.

51 Les périodes d'activité sont généralement divisées en deux parties :

- le chalutage ainsi que la recherche du poisson, actions s'étendant sur de longues durées (environ 15 heures) ;

- le débarquement du poisson qui s'effectue quant à lui plus rapidement (la durée dépassant rarement trois heures). 
Tableau 4. Répartition quotidienne entre les temps de travail et de repos du patron

\begin{tabular}{|c|c|c|c|c|}
\hline Patrons & Période & $\begin{array}{l}\text { Temps moyen de } \\
\text { repos quotidien }\end{array}$ & $\mid \begin{array}{llr}\text { Nombre } & \text { moyen } & \text { de } \\
\text { phases } & \text { de repos } \\
\text { quotidien } & & \end{array}$ & $\begin{array}{l}\text { Temps moyen de repos } \\
\text { quotidien par phase de } \\
\text { repos }\end{array}$ \\
\hline \multirow{2}{*}{ Baptiste } & juin & $6 \mathrm{~h} 23$ & 2,25 & $2 \mathrm{~h} 50$ \\
\hline & novembre & $4 \mathrm{~h}$ & 2 & $2 \mathrm{~h}$ \\
\hline \multirow{2}{*}{ Brice } & juin & $7 \mathrm{~h} 36$ & 2,4 & $3 \mathrm{~h} 10$ \\
\hline & novembre & $7 \mathrm{~h} 4$ & 2,5 & $2 \mathrm{~h} 50$ \\
\hline \multirow{2}{*}{ Benjamin } & juin & $5 \mathrm{~h} 8$ & 2,25 & $2 \mathrm{~h} 17$ \\
\hline & novembre & $6 \mathrm{~h} 23$ & 1,75 & 3 h 39 \\
\hline \multirow{3}{*}{ Total } & juin & $6 \mathrm{~h} 22$ & 2,3 & $2 \mathrm{~h} 46$ \\
\hline & novembre & 5 h 49 & 2,08 & $2 \mathrm{~h} 48$ \\
\hline & $\begin{array}{l}\text { sur } \\
\text { l'année }\end{array}$ & $6 \mathrm{~h} 5$ & 2,19 & $2 \mathrm{~h} 47$ \\
\hline
\end{tabular}

Les journées comptabilisées étaient complètes (24 h), à l'exception de celle du 18/11/04 où une heure de repos fut ajoutée (Benjamin). Une phase de repos correspond à l'arrêt de l'activité de patron pêcheur (sommeil dans sa cabine ou repas au « carré »).

À travers l'analyse de ces rythmes de vie, le déséquilibre dans la répartition quotidienne du temps de travail et du temps de repos semble manifeste. Sur 24 heures, la durée du temps de travail (Tableau 4) est supérieure à 18 heures, contre environ deux séquences de trois heures de repos (consacrées aux repas et au sommeil). Nous notons cependant une légère diminution de la plage de repos en automne, ce qui est justifié par la pêche nocturne de l'anchois à cette époque.

Verbalisations 11

Baptiste: "Quand tu travailles de nuit tu vas être plus fatigué. Par exemple, si tu travailles deux heures de nuit, tu vas être plus fatigué que deux heures de jour!» B8

Benjamin « À partir d'octobre, on peut travailler l'anchois la nuit, avant il n'y a pas. Et puis à partir de là, quand tu fais une 'petite journée' dans la journée, et bien t'essayes de te rattraper la nuit, pour compenser. » B28

53 À titre d'illustration, la séquence de travail la plus ardue s'est déroulée avec Benjamin du

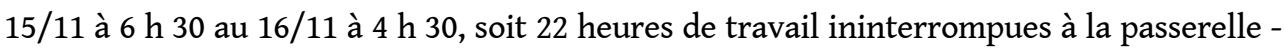
comprenant $15 \mathrm{~h} 30$ de chalutage, $4 \mathrm{~h}$ de recherche du poisson, et $2 \mathrm{~h} 30$ de route (triage du poisson par les matelots).

Parallèlement à cette description de l'activité du patron pêcheur, qui apparaît soutenue, il convient de signaler certaines périodes où les charges de travail sont moindres, voire parfois quasi inexistantes. Dans ces moments, l'ennui apparaît, et la fatigue se fait alors plus sentir chez les marins. 
Verbalisations 12

Baptiste : «Alors que c'était tranquille, tu vois c'était un peu de lassitude [...] il n'y avait plus de bateaux à côté de nous, et puis il n'y avait plus grand chose à pêcher. »

A1

Brice : "C'est reparti du moment que tu as de l'occupation, par contre, si tu te lèves et que t'as pas d'occupation [...] tu vois là, tu t'ennuies. » A14

"Quand ça pêche pas, c'est pas terrible, tu t'ennuies, t'es dans le fauteuil. » A21

Benjamin : « On a des périodes d'inactivité, de lassitude. » A28

\subsubsection{L'effet d'accumulation de fatigue}

La fatigue ne peut pas être envisagée comme un phénomène ayant des répercussions immédiates sur les patrons pêcheurs. L'étude de l'organisation du travail révèle un fort déséquilibre entre les longues périodes d'activité et les courtes périodes de repos (tableaux 3 et 4). Ceci a pour conséquence un effet de cumul de fatigue au fur et à mesure de la saison de pêche, cette accumulation devenant éprouvante en fin de saison.

- À court terme, sur 24 heures :

Verbalisations 13

Baptiste : «C'est un peu court pour moi des fois deux heures [...] après ça passe, mais je risque de ressentir un peu la fatigue dans la journée après. » A7

- À moyen terme, sur une semaine de pêche :

Verbalisations 14

Benjamin : «Les deux premiers jours de la semaine ça colle, le troisième jour ça commence à fatiguer, puis les deux derniers c'est la galère ! » A28

"C'est à partir du mercredi en général. Le mercredi on commence à payer l'addition. Trois jours d'affilée, là ça fait dimanche, lundi, mardi et puis le quatrième jour c'est mercredi [...] mercredi-jeudi c'est raide. » A30

- À long terme, sur une saison :

Au regard de la répartition des périodes annuelles de repos des marins pêcheurs (15 jours en mai et trois semaines en décembre), il apparait que la fatigue des patrons est plus prononcée à la fin du mois de novembre qu'au début du mois de juin. En outre, les conditions météorologiques difficiles et la probabilité de triage augmentent très fortement, passé le mois de septembre. D'ailleurs, les patrons différencient systématiquement la fatigue présente au début de l'été, et celle, beaucoup plus "dure », se manifestant à l'approche de l'hiver.

Verbalisations 15

Benjamin : «La fatigue qu'on peut avoir à l'heure actuelle, ça n'a rien à voir avec celle de l'automne, qui... Là, c'est de la fatigue réelle pour le corps. C'est plus dur quoi ! » A33

\subsection{Les troubles du sommeil}

\subsubsection{Les privations et le fractionnement du sommeil}

57 Les rythmes de travail et de repos sont conditionnés par la capture du poisson. En conséquence, le patron ne peut aller se coucher que lorsque le chalutier fait route (trois heures en moyenne), ou au milieu d'un " trait » de chalut (quelques minutes seulement entre les manœuvres).

Verbalisations 16

Baptiste : «Si je fais deux fois trois heures, après, je peux faire de six à dix heures sans... Alors que là, si je fais deux fois deux heures, j'ai un grand coup de barre au milieu ou vers 18 heures. » A7 
Brice: "Quand on fait vraiment la journée, la nuit il faut débarquer la pêche. Fréquemment, on dormait deux heures par jour hein! [...] Tu revenais, tu pêchais, tu pêchais, tu faisais toute la journée de triage... Tu arrivais dans le port, tu dormais toujours pas, en fait tu dormais qu'au départ. » A13

«Si on a quatre heures de route, bah moi ça me suffit pour dormir [...] donc maintenant, si tu n'as pas beaucoup de route... » A22

Benjamin : «Les grosses périodes de fatigue, où on fait une accumulation de travail et d'heures de sommeil en moins. [...] Il y a des périodes de l'année où l'on va dormir quatre heures, cinq heures on va dire, en deux fois. » A28

Ainsi, le sommeil des patrons prend place lors des trajets de départ et de retour au port, entrecoupés par le débarquement du poisson. Durant les trajets, le pilotage du navire est confié à un matelot. Les durées de sommeil sont dépendantes du contexte de pêche, car les zones de chalutage peuvent être plus ou moins éloignées des ports de débarquement (tableaux 3 et 4). Cependant, le patron peut être amené à demeurer malgré tout sur la passerelle dans deux contextes spécifiques:

- à l'aller pour localiser les bancs de poissons ;

- au retour lorsque le triage du poisson s'avère nécessaire.

\subsubsection{Les endormissements}

L'observateur embarqué a noté la survenue d'endormissements au poste de pilotage. Ces « coups de fatigue » intenses nécessitent une vigilance accrue de la part du patron, pour ne pas se laisser déborder par le sommeil.

Verbalisations 17

Baptiste : «Je piquais un peu dans l'après-midi. » A1

«Les épaules, tu les sens un peu lourdes.» A1

60 Les baisses de vigilance sont réduites en fonction de l'activité accrue du patron. En effet, lorsque les contraintes liées à l'activité de pêche sont élevées, notamment lors de l'immersion du filet (écartement avec le veau, réglages du filet selon la détection, trafic dense), la vigilance demeure élevée. À l'inverse, les périodes de moindre activité sont propices aux endormissements, principalement lors du retour au port.

Verbalisations 18

Baptiste: «Et une fois qu'on a fait la journée [...] toute cette attention, ben ça retombe. Et c'est là que tu ressens la fatigue. » $\mathrm{A} 5$

Brice : « Parce que quand tu passes les 20-30 heures bien stressé et tout, il arrive un moment où tu es obligé de te vider! Tu sais quand tu fais route terre, c'est normal [...] tout descend d'un coup! Tout le stress, tout! [...] Et là, ça peut être dangereux! »A17

\subsubsection{Une latence d'endormissement}

61 Les marins perçoivent un temps de latence de quelques minutes avant de pouvoir s'endormir. Cette durée est en relation avec la quantité de poisson pêchée. En d'autres termes, plus les patrons pêchent de poisson, plus ils auront tendance à trouver le sommeil rapidement.

Verbalisations 19

Baptiste: «Des fois je mets un quart d'heure, 20 minutes [...] parfois une demi-

heure. » A11

Brice : «La première demi-heure c'est un peu dur, mais après tu t'endors. » B17

«Je dormais pas, je tournais, je virais... Je me suis levé deux fois, j’ai regardé si

Nicolas n'était pas parti. » A16 
62 attendre la marée montante : il savait que ce retard de trois heures ne serait pas
rattrapable le lendemain, et que son bateau risquait d'arriver après les «bons coups ».

En outre, les patrons pêcheurs qui fractionnent régulièrement leur temps de sommeil en deux parties (avant et après le débarquement), avouent avoir plus de facilité à s'endormir quand le chalutier rentre au port. Ceci peut s'expliquer par deux ordres de raisons :

- lorsqu'ils rentrent au port, c'est qu'ils ont un minimum de caisses de poisson pour la vente, sinon, ils seraient restés en mer ; alors qu'à la sortie du port, les patrons vont plutôt se soucier de la journée suivante qui n'est pas assurée ;

- au départ du port la fatigue est plus importante qu'au ? retour (le patron dort lors du trajet retour).

Verbalisations 20

Baptiste : «Quand on rentre, je vais m'endormir plus vite mais quand on repart, par contre, je vais euh... pendant cinq minutes ou même une demi-heure. » A6 Benjamin : "Quand on ressort d'un port après avoir débarqué, j'ai la misère avant de dormir. Car ayant dormi trois heures... par exemple, tu vois cette nuit : j'ai dormi quatre heures, on est rentré à La Rochelle, on a débarqué ça, eh ben j'avais plus sommeil ! » A36

\subsubsection{Un sommeil plus léger}

Quand ils dorment, les patrons pêcheurs perçoivent un sommeil moins profond qu'à terre, et ce, indépendamment du confort relatif apporté par les couchettes (le patron dispose d'une couchette dans sa propre cabine).

Verbalisations 21

Baptiste : « Le sommeil est beaucoup plus profond qu'en mer. » A6

Baptiste mentionne le fait qu'en mer, deux ou trois secondes seulement lui paraissent nécessaires pour se réveiller, alors qu'à terre, cette durée semblerait s'étaler sur 30 secondes.

Verbalisations 22

Brice : «Tu dors nettement plus mal, parce que tu t'endors mal quoi, parce que tu réfléchis. » B22

Benjamin : «Tout le monde dort bien dans le bateau, mais on dort pas comme dans une maison. C'est pas du tout le même repos [...] c'est beaucoup plus léger. » A30

«T'as un sommeil plus léger. Si un gars m'appelle, aussitôt, je vais me lever parce que c'est pas normal qu'il vienne m'appeler [...] je me lève parce qu'il y a quelque chose qui va pas. » A31

Ces propos soulignent la perception d'une «inertie» du sommeil minorée durant les moments de repos dans la couchette. Selon eux, la nécessité d'être opérationnel immédiatement est reflétée par la légèreté de leur sommeil à bord, bien que l'efficacité de la récupération ne soit pas réellement remise en cause.

\subsubsection{Un sommeil contraint}

67 Afin de différencier le sommeil du marin de celui du « terrien », il convient de prendre en compte le contexte de travail du chalutier pélagique. En effet, dans cet environnement contraignant, le confort dépend avant tout de la météo et de ses répercussions sur les mouvements du bateau.

Verbalisations 23

Baptiste: «Avec le mauvais temps je n'ai pas pu dormir en mer quoi! À taper 
comme ça, mon sommeil était assez léger. Donc après, ben quand tu fais 24 heures derrière, ça, c'est plus raide ! » $\mathrm{A} 39$

Brice : « Tu as toujours des secousses. Tu ne peux pas dormir bien. Tu peux dormir un quart d'heure, mais la première secousse va te réveiller ! » B13

Benjamin : "T'as du repos, mais tu dors pas parce que tu es toujours en train de te caler et tu somnoles. C'est pas un vrai sommeil ! » B37

De plus, les bateaux sont très bruyants : le volume sonore est constamment supérieur à 80 décibels. Le moteur fonctionne $24 \mathrm{~h} / 24 \mathrm{~h}$ car cette production d'énergie est nécessaire à la multitude d'appareils présents à bord. Cependant sur certains chalutiers, le moteur de la pompe hydraulique (entre autres le fonctionnement des treuils) est plus "agressif » pour l'audition que le moteur assurant la propulsion.

\section{Discussion}

\subsection{L'accumulation de la fatigue}

$69 \mathrm{Au}$ final, les perceptions de fatigue des marins et l'analyse des rythmes de travail semblent révéler une accumulation de fatigue des marins pêcheurs au fur et à mesure de la saison, connaissant son paroxysme en novembre (fin de saison). En premier lieu, les troubles de sommeil observés à bord des chalutiers ne faciliteraient pas la récupération de l'organisme du patron pêcheur. En second lieu, l'interruption de l'activité lors des escales ne serait pas suffisante pour récupérer de la semaine de pêche. Cette suspension de l'activité pendant le week-end dure environ 30 heures. Elle s'opère du vendredi aprèsmidi (après le nettoyage du bateau) au samedi soir (départ dans la nuit pour être sur la zone de pêche le dimanche à l'aube). Il est constaté dans nos observations que ces escales ne sont pas propices au repos, car les marins pêcheurs planifient la maintenance du navire et les diverses réparations à effectuer. Mais surtout, ils ont coutume de se divertir à terre le vendredi soir jusqu'à tard dans la nuit dans les établissements nocturnes (bars et discothèques), la consommation d'alcool pouvant être importante durant ces festivités.

70 En matière de préconisations, les problèmes de fatigue sont causés par un manque d'organisation selon Mion et Ricouard (2007). Ces auteurs recommandent, dans le cas des professions à risques, une durée de sommeil équivalente à cinq heures la nuit précédente, ou 12 heures sur les 48 heures précédentes avant de débuter l'activité à risques. Ces généralisations apparaissent limitées en ce qui concerne les chalutiers. Des pistes de régulation spécifiques, appuyées sur la connaissance de l'activité, paraissent à privilégier, dans l'intention de répondre au plus près aux attentes.

71 Dans un premier temps, il s'agit d'optimiser les périodes intermédiaires de repos tant d'un point de vue quantitatif que qualitatif. Il faudrait avant tout limiter les festivités durant l'escale. Si ces distractions donnent lieu à une certaine « évasion » psychologique et physique par rapport à l'espace confiné du navire, il ne faut pas pour autant en abuser. De plus, lorsque le départ s'effectue dans la nuit de samedi à dimanche, le fait de décaler le départ à $6 \mathrm{~h}$ par exemple, offrirait une nuit supplémentaire de repos à terre. Ceci garantirait une récupération bien meilleure (deux nuits d'escale au lieu d'une), tout en n'ôtant que quelques heures à l'activité de pêche.

72 Dans un second temps, certains équipages enrôlant deux matelots supplémentaires afin d'effectuer une rotation du personnel (repos à terre), il serait peut-être envisageable d'enrôler un patron salarié pour une ou deux paires de chalutiers : ce dans l'intention 
de libérer une semaine de repos, au cours d'un mois de travail. Cette plage de repos mensuelle pourrait en outre offrir l'occasion d'une mise à jour du travail administratif. Benjamin avait d'ailleurs recours à cette solution, moins contraignante pour lui du fait du remboursement de sa paire de chalutiers.

\subsection{La charge de travail du patron pêcheur}

Hormis le point de vue macroscopique des rythmes de travail éprouvants imposés aux chalutiers, c'est la micro-analyse de la charge de travail qui fait ressortir plus finement les causes possibles de la fatigue du patron pêcheur.

À la passerelle, son activité comprend des tâches multiples :

- surveiller les différents appareils électroniques, ainsi que les voyants d'alarme du moteur ; d'après Jegaden (2001), cette activité consiste à maintenir un niveau de vigilance constant sur ces systèmes robotisés ;

- assurer le pilotage du navire, et diriger les manœuvres de l'équipage et du veau (contraintes pour les chalutiers pélagiques qui travaillent en paire);

- communiquer avec son équipage, son veau, ainsi qu'avec les autres bateaux - notamment pour détecter et suivre le poisson, mais aussi avec la «terre » (criée, mareyeur, matelot de repos, etc.).

Cette multitude de tâches à effectuer dans un laps de temps parfois extrêmement court pose problème au patron : il est obligé de sélectionner les informations pertinentes, et corrélativement, de reléguer au second plan les données moins essentielles. Cette concentration perceptuelle sur un domaine précis se traduit par une déperdition cognitive relative à d'autres sources d'information (Itti et coll., 2005).

Les contraintes associées à la charge de travail à bord des chalutiers seraient l'afflux simultané d'informations concernant la conduite de la paire de navires, le déplacement du poisson, le positionnement des autres bateaux... Ces données nécessitent une analyse permanente de la part du patron: à chaque catégorie d'information correspond une interprétation visant à évaluer objectivement la situation représentée par l'écran de radar ou la communication radio (Hazelhurst, 1999).

En ce qui concerne les astreintes, nous relevons en priorité la vigilance permanente nécessitée comme origine possible de la fatigue du patron pêcheur. D'ailleurs, celle-ci entraîne dans certaines situations une "surcharge mentale " (Jourdan et coll., 2002). Le cas échéant, le patron pêcheur ne prendra aucun risque; il programmera alors ses différentes actions en fonction de leurs urgences temporelles respectives. Il réalisera une hiérarchisation entre les différents " attracteurs cognitifs ». À titre d'exemple, le patron va ainsi dévier la trajectoire du navire pour éviter une collision, même s'il doit s'écarter momentanément du poisson (détection au sonar), et à cet instant, il va interrompre sa conversation à la $\mathrm{CB}$ pour la reprendre ultérieurement. De par son expertise des situations à risque, le patron pêcheur va savoir s'adapter aux conditions pour maintenir un niveau de sécurité maximal (Morel et coll., 2008).

78 En matière de préconisation, il serait bénéfique du point de vue de la vigilance d'entrecouper les longues séquences d'activité mentionnées dans les observations et verbalisations (environ 16 heures consécutives). Il s'agirait de généraliser une brève phase de sommeil afin de scinder l'activité en deux périodes. Cette sieste pourrait s'inspirer du sommeil caractéristique des skippers en solitaire, c'est-à-dire de courte 
durée et à un moment propice au sommeil (Chauve et Catala, 2002). Ainsi, la recommandation de 20 minutes de repos (isolé dans sa cabine) devrait s'imposer au quotidien après le déjeuner. Cet horaire favoriserait une faible latence d'endormissement, tandis que la durée de 20 minutes n'entraînerait pas une forte inertie du sommeil. Cette solution n'était envisagée jusque-là qu'en cas de fatigue extrême du patron.

\subsection{Les enjeux de cette étude} les marins pêcheurs. Elle a pour objectif de décrire l'activité des marins et se veut donc centrée sur la validité individuelle. Leplat $(2002,2008)$ utilise le terme d' «étude de cas » pour définir ces recherches répondant à une démarche compréhensive, et qui dotent d'« un caractère approfondi » l'analyse de l'activité.

ce sens, notre étude, basée sur un nombre restreint de sujets, n'a pas la prétention d'avoir une portée de généralisation. Néanmoins, les cas analysés ne sont pas isolés ou exceptionnels. Les résultats proviennent d'une certaine convergence interindividuelle entre les trois sujets. Ces études de terrain privilégient plutôt la validité interne entre les acteurs et l'environnement, au détriment de cette portée de généralisation.

81 Cette validité est renforcée par la démarche méthodologique employée - le recueil de données en contexte. Cette investigation sur le terrain a d'ailleurs constitué un enjeu majeur de cette étude puisque l'environnement du chalutier est difficilement accessible, tant d'un point de vue physique que social. Effectivement, la proximité entre le chercheur et ses sujets est alors réduite à sa plus simple expression, ne serait-ce qu'en fonction de l'espace restreint et du nombre de jours passés en mer sans interruption (Maline et Dorval, 1991a). Dans l'environnement du bateau, la situation d'observation ethnographique est portée à son paroxysme, il est même impossible pour le chercheur de sortir de la situation (Duval, 1998). Pour contourner cette difficulté, l'observateur a dû gagner la confiance des différents membres d'équipages en passant "l'épreuve » de la phase de familiarisation. C'est-à-dire que l'observateur devait faciliter son insertion dans l'environnement d'étude, par ses connaissances, mais aussi par son comportement propre. Une expérience préalable de l'embarquement maritime était ici indispensable. Effectivement, cette intégration parmi les gens de mer nécessitait de respecter les règles implicites de ce milieu, réclamant une certaine retenue dans le comportement. Par exemple, il s'agissait d'attendre le moment opportun pour le questionnement, quitte à ne pas trop parler pendant les premiers jours passés en mer.

\section{Conclusion}

Cette étude de la fatigue des patrons pêcheurs adopte une perspective ergonomique à travers la description et l'analyse de leur activité quotidienne en " milieu naturel » - à bord des chalutiers. Selon nous, la caractérisation de cette fatigue s'avère utile pour optimiser la sécurité à bord des navires, notamment la prévention des endormissements susceptibles d'engendrer un défaut de veille. Notre approche a combiné le recueil des perceptions subjectives des marins, sous forme d'observations et sous forme d'entretiens. La fatigue est avant tout causée par les rythmes de travail épuisants des patrons pêcheurs. L'analyse de la fatigue perçue met en évidence l'existence d'un phénomène de

Perspectives interdisciplinaires sur le travail et la santé, 13-1 | 2011 
cumul tout au long de la saison de pêche (début juin à début décembre), que les périodes de récupération ponctuelles ne parviennent à pallier. De même, l'on peut supposer que les troubles du sommeil observés durant les embarquements ne facilitent pas cette récupération.

En outre, l'analyse de l'activité du patron pêcheur fait ressortir la multiplicité des tâches à réaliser (manœuvres du train de pêche, réglages du filet, pilotage du bateau, recherche du poisson, les communications, la surveillance des appareils électroniques...). Elles sont effectuées dans une fenêtre temporelle restreinte, avec le stress et les risques qui en découlent.

En définitive, ces résultats soulignent l'intérêt de poursuivre les recherches dans le domaine de l'ergonomie maritime malgré les contraintes fortes rencontrées sur ce type de terrain d'étude (Minguy et coll., 1998). En référence à cette problématique de la fatigue, il serait intéressant à l'avenir de confronter des mesures physiologiques aux données de la fatigue perçue (Sennegon et coll., 2009). Ceci autoriserait une approche globale de la fatigue sur ces deux plans d'investigation distincts, physiologique et psychologique, qui entretiennent des relations causales non univoques. D'autant plus que des décalages sont repérables, entre la fatigue physiologique d'un organisme d'une part, et la perception qu'en aurait l'individu d'autre part. Ces décalages pouvant être potentiellement à la source d'incidents ou d'accidents.

\section{BIBLIOGRAPHIE}

Andro, M., Dorval, P., Le Bouar, G., Le Pluart, C., Le Roy, Y., Roullot, C., Meillat, M., Prado, J. (1987). Sécurité et conditions de travail à la pêche artisanale et semi industrielle. Brest : IFREMER.

Bachelor, A., Joshi, P. (1986). La méthode phénoménologique de recherche en psychologie. Québec, Presses de l'Université Laval.

Becker, H.S. (1958). Problems of inference and proof in participant observation. American Sociological Review, 23, 652-666.

Benoit, O., Foret, J. (1992). Le sommeil humain - bases expérimentales physiologiques et physiopathologiques. Paris, Masson.

Boivin, D.B., James, F.O., Casademont, A. (2005). L'exposition contrôlée à la lumière et à l'obscurité ajuste le rythme du cortisol salivaire chez les travailleurs de nuit. PISTES, 7, 2. https:// journals.openedition.org/pistes/3200

Bureau Enquêtes Accidents (2007). 1997 - 2007, Dix ans d'expérience, d'enquêtes, d'études et de recommandations de sécurité. www.beamer-france.org/BanqueDocument/pdf_34.pdf

Cespuglio, R., Bonnet, C., Faradji, H., Buguet, A. (1986). Stress et sommeil : données phénoménologiques et mécanismes centraux chez l'animal. Dans, Actes INRETS, 79, 51-55.

Chauve, J.Y., Catala, I. (2002). La course en solitaire. Bulletin Veille-Sommeil, 11-14. 
Chrousos, G.P., Gold P., W. (1992). In I. Paulhan et M. Bourgeois, (1995). Stress et coping : les stratégies d'ajustement à l'adversité (pp. 14-20). Paris : Presses Universitaires de France.

Direction générale de la mer et des transports (2007). Rapports et statistiques 2007 : Les accidents du travail maritime. www.developpement-durable.gouv.fr/IMG/spipdgmt/pdf/yd-1136couverture_stat1_2007.pdf_-_Adobe_Acrobat_Professional_cle7771ff.pdf

Duval, M. (1998). Ni morts, ni vivants : marins! - Pour une ethnologie du huis clos. Paris, Presses Universitaires de France.

Gander, P., Van Den Berg, M., Signal, L. (2008). Sleep and sleepiness of fishermen on rotating schedules. Chronobiology International, 25, 2, 389-398.

Geslin, P. (2002). Les formes sociales d'appropriations des objets techniques, ou le paradigme anthropotechnique. Ethnographiques.org, 1. www.ethnographiques.org/2002/Geslin.html Gold, R.L. (1958). Roles in sociological field observations. Social Forces, 36, 217-223.

Grison, B., Riff, J. (2002). Validité écologique et situations d'étude privilégiées : de la Psychologie Expérimentale à l'Anthropologie Cognitive Située. Dans, Actes des quatrièmes journées d'études de l'association ACT'ING, Objets théoriques objets de conception objets d'analyse et situations d'étude privilégiées, Domaine de Chalès.

Gunnarsson, L.G., Bäck, H., Jones, I., Olsson, T. (2004). Stress recovery during an ocean boat race. Stress and health, 20, 165-171.

Hazelhurst, B. (1999). Fishing for cognition : an ethnography of fishing practice in a community on the west coast of Sweden. Thèse de Doctorat non publiée, University of California, San Diego.

Itti, L., Rees, G., Tsotsos, J.K. (2005). Neurobiology of Attention. London, San Diego : Elsevier Academic Press.

Jegaden, D. (2001). Le métier de marin : grandeur et paradoxes. Dans, Actes du Colloque Mer \& Santé 20 septembre 2001, Brest.

Jourdan, M., Theureau, J. (2002). Charge mentale : notion floue et vrai problème. Toulouse, Octarès. Jouvet, M. (2000). Le sommeil et le rêve. Paris, Odile Jacob.

La Giclais, B. de (2009). Le sommeil particulier des navigateurs solitaires à la voile en course transocéanique pour une bonne vigilance. Dans, Quatorzièmes journées de la médecine des gens de mer. Paris. www.developpement-durable.gouv.fr/14emes-journees-de-la-medecine-des.html

Laperrière, A. (1992). L'observation directe. Dans, B. Gauthier, Recherche Sociale : de la problématique à la collecte des données (pp. 251-272), Sillery, Presses de l'Université du Québec.

Laplantine, F. (1996). La description ethnographique. Paris, Nathan.

Lavoie, S., Paquet, P., Selmaoui, B., Rufiange, M., Dumont, M. (2003). Vigilance levels during and after bright light exposure in the first half of the night. Chronobiology International, 20, 6 , 1019-1038.

Leplat, J. (2000). L'analyse psychologique de l'activité en ergonomie. Toulouse, Octarès.

Leplat, J. (2002). De l'étude de cas à l'analyse de l'activité. PISTES, 4, 2. https://

journals.openedition.org/pistes/3658

Leplat, J. (2008). Repères pour l'analyse de l'activité en ergonomie. Paris, Presses Universitaires de France. 
Le Roy, Y., Breuille, P. (1997). La fiabilité humaine dans la sécurité en mer. Dans, Troisièmes journées de la médecine des gens de mer. Paris.

Le Scanff, C. (2000). Les aventuriers de l'extrême. Paris : Calmann-Lévy.

Maline, J., Dorval, P. (1991a). Analyse du travail d'un équipage : prise en compte de l'activité des marins pêcheurs. Le travail humain, 54, 2, 165-184.

Maline, J., Dorval, P. (1991b). Analyse de travail du marin pêcheur et fréquence cardiaque. Archives des maladies professionnelles de médecine du travail et de sécurité sociale, 52, 1, 7-15.

Mion, G., Ricouard, S. (2007). Repos de sécurité : quels enjeux ? Annales Françaises d'Anesthésie et de Réanimation.

Minguy, J., Chauvin, C. (1998). Recherche en ergonomie des activités maritimes. Dans, Actes du colloque Recherche et Ergonomie. Toulouse.

Morel, G., Amalberti, R., Chauvin, C. (2008). Safety and Resilience : Articulation and Difference The example of the professionnal fishing industry. Human Factors, 50, 1, 1-16.

Saper, C.B., Scammel, T.E., Lu, J. (2005). Review Article Hypothalamic regulation of sleep and circadian rhythms. Nature, 437, 1257-1263.

Sennegon, B., Grison, B., Buttelli, O. (2009). Les Processus de fatigue en milieu maritime, approche psychophysiologique. Dans, Quatorzièmes Journées de la médecine des gens de mer, Paris. www.developpement-durable.gouv.fr/14emes-journees-de-la-medecine-des.html Sennegon, B., Grison, B., Buttelli, O. (2009). Les Processus de fatigue des skippers en solitaire, approche psychophysiologique. Dans, XIIIème Congrès International ACAPS, Lyon.

Siegel, J. (2002). The neural control of sleep and waking. New York, Wien, Springer Verlag.

Stampi, C. (1989).Ultrashort sleep / Wake patterns and Sustained Performance. In D.F. Dinges, R.J. Broughton, Sleep an Alertness: Chronobiological, Behavioral, and Medical Aspects of Napping (pp. 139-167), New York, Raven Press.

Theureau, J., Jeffroy, F. (1994). Ergonomie des situations informatisées. Toulouse, Octarès.

Theureau, J. (2004). L'hypothèse de la cognition (ou action) située et la tradition d'analyse du travail de l'ergonomie de langue française. @ctivités, 1, 2, 11-25. http://activites.org/v1n2/ theureau.pdf

Theureau, J. (2006). Le cours d'action : méthode développée. Toulouse, Octarès.

Tirilly, G., Foret, J. (2000). Évolution du rythme activité/repos et de la vigilance subjective d'un équipage de marins pêcheurs. Dans, Actes du Congrès de la SELF 2000.

Vermersch, P. (2000). Conscience directe et conscience réfléchie. Intellectica, 2, 31, 269-311.

\section{RÉSUMÉS}

Nous avons analysé les processus de fatigue à travers l'activité de trois patrons pêcheurs de chalutiers pélagiques.

Nous nous sommes focalisés sur la fatigue perçue d'un point de vue psychologique. Le recueil de données s'est effectué en situation naturelle. Notre méthode a combiné l'observation directe et des entretiens compréhensifs. Les rythmes de travail sont la principale cause de fatigue, car les périodes de repos n'autorisent pas une récupération suffisante. Ainsi, nous notons une accumulation de la fatigue d'ordre psychologique tout au long de la saison de pêche. Cette 
récupération est limitée par les troubles du sommeil observés à bord. De surcroît, l'analyse de l'activité du patron pêcheur révèle une charge de travail conséquente à la source de stress et de risque dans ce contexte de vigilance minorée.

We analyzed the dynamics of the fatigue processes through the activity of pelagic trawlers captains.

We focussed on perceived fatigue from a psychological point of view. The data were collected in natural situation. Our methodology combined direct observation with non-directive interviews.

The work rhythms aboard trawlers (phases of work and rest alternation) are the main cause of the captain's fatigue. The short periods of rest do not authorize a sufficient recovery. Thus, we note a fatigue accumulation throughout the trawling season. In addition, the recovery is restricted by the sleep disorders observed during the embarkations. The analysis of the trawler captain's activity reveals workload, causing stress, as well as risk-taking in a diminished vigilance context.

Analizamos los procesos de fatiga a traves de la actividad de tres capitanes de pesca de los barcos pescadores pelágicos. Nos concentramos sobre la fatiga percibida desde el punto de vista psicológico. La colecta de datos se efectuó en situacion natural. Nuestro método combina la observación directa y entrevistas no dirigidas. Los ritmos de trabajo son la principal fuente de fatiga porque los periodos de reposo no permiten una recuperación suficiente. Así, notamos una acumulación de la fatiga psicológica a lo largo de la estación de pesca. La recuperación esta limitada por problemas de insomnio observados a bordo. El análisis de la actividad del capitán pescador revela una carga de trabajo que es fuente de estrés y de riesgo en el contexto de una capacidad de vigilancia reducida.

\section{INDEX}

Keywords : pelagic trawlers, fatigue, activity, workload, work rhythms

Palabras claves : barcos pescadores pelágicos, fatiga, actividad, carga de trabajova, ritmos de trabajo

Mots-clés : chalutiers pélagiques, fatigue, activité, charge de travail, rythmes de travail

\section{AUTEURS}

\section{BASTIEN SENNEGON}

bastiensennegon@yahoo.fr, Faculté du sport et de l'éducation physique, rue de Vendôme -BP 6237- 45062 Orléans cedex 2

\section{BENOIT GRISON}

benoit.grison@univ-orleans.fr, Faculté du sport et de l'éducation physique, rue de Vendôme -BP 6237- 45062 Orléans cedex 2

\section{PHILIPPE RAVIER}

philippe.ravier@univ-orleans.fr, Polytech Orléans, 12 rue de Blois -BP 6744- 45067 Orléans cedex 2 


\section{OLIVIER BUTTELLI}

olivier.buttelli@univ-orleans.fr, Faculté du sport et de l'éducation physique, rue de Vendôme -BP 6237- 45062 Orléans cedex 2 